\title{
New strategies of urban sports culture development urbanization process
}

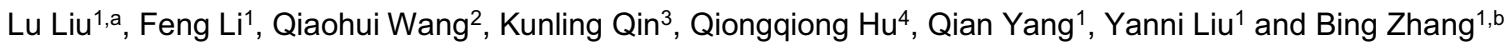 \\ ${ }^{1}$ Institute of Physical Education, Huanggang Normal University, Huangzhou 438000, China \\ ${ }^{2}$ Macaomiao Primary School, Tuanfeng 438000, China \\ ${ }^{3}$ Yidu Gaobazhou Middle School, Yidu 443300, China \\ ${ }^{4}$ Fangxian Mengu Middle School, Shiyan 442108, China
}

\begin{abstract}
Sports culture demand continues to increase, to the reasonable development, it is necessary to establish the development system of comprehensive evaluation on the system. In this paper, the fuzzy mathematics method is applied to China's new urbanization of small and medium-sized town sports culture development strategy has carried on the comprehensive evaluation, which for the determination of weight, also adopted the method of analytic hierarchy process (ahp) to determine the impact factors, and through the inspection of mathematical statistics, finally reached a conclusion that China's new urban sports culture development strategy of urbanization level of evaluation for good, proved that China's new urbanization of small and medium-sized town sports culture development strategy generally applaud but still exist deficiencies, needs further perfection and development of the society from all walks of life.
\end{abstract}

\section{Introduction}

China with 13 hundred million population is a great agricultural country, from which nearly0.7 times people are rural people, with the 17 th $\mathrm{CPC}$ national congress succeeded hosting, " Three rural issues "was regarded as key object in national development, because it related to China future development, and was closely related to improve rural living quality, with rural living quality improvements, people demands on health were also constantly deepening, just in such backgrounds, China new pattern urbanization process medium and small towns sports application emerged, rural sports development was town-oriented, took peasants sports association as bond, with villagers neighborhood committee as basis, and then formed radioactive organizational network model, therefore it could really set up ideas of adjusting measures to local conditions and scientific development, which did favor to massive peasants.

Regarding China new pattern urbanization process medium and small towns sports cultural correlation articles, they are emerging in endlessly, main social problems, small towns sports, work on developing China new pattern urbanization process medium and small towns sports is an important indicator to establish small towns, for such similar articles, formers have made lots of efforts, such as in the beginning of the century, China worked on researching on new pattern urbanization process's medium and small towns sports development, medium and small towns influential development factors, medium and small towns sports communities and sports cultural development trend, medium and small towns sports social insurance as well as medium towns sports cultural construction theory frame construction and other problems, so it generated current China towns sports being full of passions and vigor; $\mathrm{Xu}$ Feng in the article regarding masses sports demands orientation and development countermeasures, by applying documents literature, mathematical statistics, questionnaire survey and other methods to make analysis and research on one region masses sports social demands, cultural demands, personal demands and ecological requirement as well as others each factor, finally got the region masses sports demands powerful impetus was due to people body and heart building demands as well as pursuit and yearning colorful life, and advocated that masses should positive participate in physical exercises, sports consumption from them, establish a diversified community sports network, strengthen sports fitness apparatus development, and meanwhile should constantly advertise relative knowledge to establish a reasonable, effective community sports service system.

The paper just on the former researches basis, makes further analysis and research on China new pattern urbanization process medium and small towns sports culture development, links theory with practice, based on the principle of seeking truth from fact, it makes efforts in China new pattern urbanization process medium and small towns sports culture development aspect researching.

\section{Comprehensive evaluation on new pattern urbanization process medium}

a, b Corresponding author: ${ }^{\mathrm{a}}$ Ty-11@hgnu.edu.cn;

$$
\text { b tiyuxi@qq.com }
$$




\section{and small towns sports culture development strategy}

In town sports development process, constantly expand sports population ratio on whole population has important significances, and is also an important mark to measure a social civilization development, sports culture is quintessence of them, therefore sports cultural research is beneficial to sports rapid development, so it has important significances.

In order to more objective, air, practical evaluate China new pattern urbanization process medium and small towns sports culture development strategies, the paper adopts fuzzy mathematical methods, its principle is as following show.

\section{Fuzzy evaluation application}

To new pattern urbanization process medium and small towns sports culture development strategies system selected partial factors, it makes analysis, as following Table 1 shows: By above theory, establish evaluation set that:

$V=\left\{\mathrm{v}_{1}, \mathrm{v}_{2}, \mathrm{v}_{3}, \mathrm{v}_{4}, \mathrm{v}_{5}\right\}=\{$ exce1lent, good, general, poor, bad $\}$ After that, for above every factor, it makes judgment, and meanwhile applies analytic hierarchy process to align weights, its principle is as following.

\section{Analytic Hierarchy Process theoretical model}

AHP features are hierarch zing complicated problems, making clear about primary and secondary, possessing stronger logicality and hierarchical structure, the algorithm mainly is calculating indicators weights. It is applicable to comprehensive assessment system, is a powerful mathematical method that converts problems into quantitative research. Nowadays analytic hierarchy process has already widely used in each field to solve practical problems. China new pattern urbanization process medium and small towns sports culture development strategies comprehensive assessment involves multiple reference indicators, the decision problems is suitable to analytic hierarchy process.

\section{Application of analytic hierarchy process}

According to above process, it can get corresponding judgment matrix, apply above theory, Combine with above factors and link with one company specific status; it gets each indicator evaluation results, as following

Table 2 show:

By above table, it can make fuzzy comprehensive evaluation, combine with previous stated formula, it can get its first grade fuzzy evaluation result, as following Table 3 show:

Table 1. New pattern urbanization process's medium and small towns' sports culture development strategies influence factors.

\begin{tabular}{|c|c|c|}
\hline \multirow{19}{*}{$\begin{array}{l}\text { New pattern urbanization } \\
\text { process's medium and small towns } \\
\text { sports culture development } \\
\text { strategies factors U }\end{array}$} & \multirow{4}{*}{ Subjective factor $\left(U_{1}\right)$} & Mass self quality $\left(U_{11}\right)$ \\
\hline & & Home conditions $\left(U_{12}\right)$ \\
\hline & & Living way $\left(U_{13}\right)$ \\
\hline & & Values $\left(U_{14}\right)$ \\
\hline & \multirow{5}{*}{ Objective factor $\left(U_{2}\right)$} & Community organizations $\left(U_{21}\right)$ \\
\hline & & Existing facilities $\left(U_{22}\right)$ \\
\hline & & Natural resources $\left(U_{23}\right)$ \\
\hline & & Household economy $\left(U_{24}\right)$ \\
\hline & & Surroundings $\left(U_{25}\right)$ \\
\hline & \multirow{3}{*}{ Internal impetus $\left(U_{3}\right)$} & Internal environment $\left(U_{31}\right)$ \\
\hline & & Structural impetus $\left(U_{32}\right)$ \\
\hline & & Resource allocation $\left(U_{33}\right)$ \\
\hline & \multirow{2}{*}{ Realistic conditions $\left(U_{4}\right)$} & Mass media $\left._{U_{41}}\right)$ \\
\hline & & Government sector $\left(U_{42}\right)$ \\
\hline & \multirow{5}{*}{ External impetus $\left(U_{5}\right)$} & External environment $\left(U_{51}\right)$ \\
\hline & & Market factor $\left(U_{52}\right)$ \\
\hline & & Western culture $\left(U_{53}\right)$ \\
\hline & & Chinese traditional culture $\left(U_{54}\right)$ \\
\hline & & Organization management $\left(U_{55}\right)$ \\
\hline
\end{tabular}


Table 2. Each indicator evaluation result.

\begin{tabular}{|c|c|c|c|c|c|c|c|}
\hline \multirow{21}{*}{$\mathrm{U}$} & \multirow{2}{*}{$\begin{array}{c}\text { First grade } \\
\text { indicator } \\
\left(U_{\mathrm{i}}\right)\end{array}$} & \multirow{2}{*}{$\begin{array}{l}\text { Second grade } \\
\text { indicator } \\
\left(U_{\mathrm{ik}}\right)\end{array}$} & \multicolumn{5}{|c|}{ Evaluation value $F_{\mathrm{ikj}}$} \\
\hline & & & $\begin{array}{c}\text { Excelle } \\
n t\end{array}$ & Good & General & Poor & $\mathrm{Bad}$ \\
\hline & \multirow{4}{*}{$\begin{array}{c}U_{1} \\
(0.0400)\end{array}$} & $U_{11}(0.1168)$ & 0.10 & 0.65 & 0.25 & 0.00 & 0.00 \\
\hline & & $U_{12}(0.1044)$ & 0.00 & 0.70 & 0.05 & 0.25 & 0.00 \\
\hline & & $U_{14}(0.3894)$ & 0.00 & 0.10 & 0.55 & 0.35 & 0.00 \\
\hline & & $U_{14}(0.3894)$ & 0.00 & 0.60 & 0.05 & 0.35 & 0.00 \\
\hline & \multirow{5}{*}{$\begin{array}{c}U_{2} \\
(0.0788)\end{array}$} & $U_{21}(0.0594)$ & 0.10 & 0.20 & 0.65 & 0.00 & 0.00 \\
\hline & & $U_{22}(0.0594)$ & 0.20 & 0.10 & 0.70 & 0.00 & 0.00 \\
\hline & & $U_{23}(0.1399)$ & 0.10 & 0.25 & 0.65 & 0.00 & 0.00 \\
\hline & & $U_{24}(0.4455)$ & 0.00 & 0.80 & 0.10 & 0.10 & 0.00 \\
\hline & & $U_{25}(0.2958)$ & 0.00 & 0.50 & 0.10 & 0.40 & 0.00 \\
\hline & \multirow{3}{*}{$\begin{array}{c}U_{3} \\
(0.1616)\end{array}$} & $U_{31}(0.1637)$ & 0.15 & 0.20 & 0.65 & 0.00 & 0.00 \\
\hline & & $U_{32}(0.2973)$ & 0.00 & 0.70 & 0.15 & 0.15 & 0.00 \\
\hline & & $U_{33}(0.5390)$ & 0.00 & 0.85 & 0.05 & 0.10 & 0.00 \\
\hline & \multirow{2}{*}{$\begin{array}{c}U_{4} \\
(0.3598)\end{array}$} & $U_{41}(0.5000)$ & 0.00 & 0.70 & 0.20 & 0.10 & 0.00 \\
\hline & & $U_{42}(0.5000)$ & 0.00 & 0.75 & 0.15 & 0.10 & 0.00 \\
\hline & \multirow{5}{*}{$\begin{array}{c}U_{5} \\
(0.3598)\end{array}$} & $U_{51}(0.0688)$ & 0.20 & 0.10 & 0.70 & 0.00 & 0.00 \\
\hline & & $U_{52}(0.1707)$ & 0.10 & 0.15 & 0.75 & 0.00 & 0.00 \\
\hline & & $U_{53}(0.2695)$ & 0.00 & 0.65 & 0.10 & 0.25 & 0.00 \\
\hline & & $U_{54}(0.2695)$ & 0.00 & 0.55 & 0.35 & 0.10 & 0.00 \\
\hline & & $U_{55}(0.0688)$ & 0.00 & 0.85 & 0.10 & 0.05 & 0.00 \\
\hline
\end{tabular}

Table 3. First grade fuzzy comprehensive evaluation table.

\begin{tabular}{|c|c|c|c|c|c|c|}
\hline \multirow{3}{*}{$U_{\mathrm{i}}$} & \multicolumn{5}{|c|}{ Evaluation value $\mathrm{b}_{\mathrm{ij}}$} \\
\cline { 3 - 7 } & & Excellent & Good & General & Poor & Bad \\
\cline { 2 - 7 } & $U_{1}$ & 0.0177 & 0.5501 & 0.1396 & 0.2987 & 0.0000 \\
\cline { 2 - 7 } & $U_{2}$ & 0.0348 & 0.5571 & 0.2453 & 0.1629 & 0.0000 \\
\cline { 2 - 7 } & $U_{3}$ & 0.0246 & 0.6990 & 0.2780 & 0.0985 & 0.0000 \\
\cline { 2 - 7 } & $U_{4}$ & 0.0000 & 0.7250 & 0.1750 & 0.1000 & 0.0000 \\
\cline { 2 - 7 } & $U_{5}$ & 0.0308 & 0.6044 & 0.2694 & 0.0954 & 0.0000 \\
\hline
\end{tabular}

After that, combine with previous formula, it makes second grade evaluation, that:

$B_{i}=A \circ R=\left(\mathrm{a}_{1}, \mathrm{a}_{2}, \cdots \mathrm{a}_{5}\right) \circ\left(\mathrm{B}_{1}, \mathrm{~B}_{2}, \cdots \mathrm{B}_{5}\right)^{T}=(0.0813,0.8573,0.0136,0.0149,0.0000)$

According to maximum membership principle, it can get that 0.85613 is final evaluation result.

\section{Conclusion}

The paper mainly discusses China new pattern urbanization process's medium and small towns sports culture development strategy influence factors, and applies fuzzy mathematical method to make systematic and objective evaluation on China new pattern urbanization process's medium and small towns sports culture development strategy, from which by applying analytic hierarchy process method, it defines China new pattern urbanization process's medium and small towns sports culture development strategy weights, finally by second grade evaluation, it gets China new pattern urbanization process's medium and small towns sports culture development strategy evaluation is 0.85613 that belongs to good level, but it still has something to be improved, so the writer suggests government sectors should strengthen sports cultural advertising to build good basis for China's tows sports cultural development.

\section{Acknowledgment}

This research was supported by Huanggang Normal University in 2015 research projects, the personalized teaching aerobics study (2015CE18). 2014 Hubei province project in humanities and social science: in the construction of urbanization path of the development of rural school sports research (14G383). 2015 Huanggang normal university research projects, rural school sports development path, based on the perspective of government purchasing public sports services (2015017003). 


\section{References}

1. Qi Wei-Ping, Journal of Henan normal university, 32, 59-61(2005).

2. Wan Hao, Journal of physical education, 8, 2023(2001).

3. Gong De-Gui, Journal of physical education, 12, 4447(2005).
4. Zhao Jun, Xiao Hong-An, Agricultural modernization research, 25, 22-26(2004).

5. Li Song-Zhi, Journal of geographical environment in Yunnan, 13, 21-25(2001).

6. Z. L. Liu, Journal of Applied Sciences, 13, 47024707(2013).

7. Cai G, Yao D, Sun J, et al. Journal of Coastal Research, 73, 792-796(2015). 